Recherches en didactique des langues et des cultures

Les cahiers de l'Acedle

$13-2 \mid 2016$

Interactions langagières et didactique des langues

\title{
La récapitulation en activité d'expression orale : entre complexité et autonomie
}

Farid Mazi

\section{OpenEdition}

Journals

Édition électronique

URL : http://journals.openedition.org/rdlc/916

DOI : $10.4000 /$ rdlc. 916

ISSN : 1958-5772

Éditeur

ACEDLE

Référence électronique

Farid Mazi, «La récapitulation en activité d'expression orale : entre complexité et autonomie ",

Recherches en didactique des langues et des cultures [En ligne], 13-2 | 2016, mis en ligne le 25

septembre 2016, consulté le 01 mai 2019. URL : http://journals.openedition.org/rdlc/916 ; DOI :

$10.4000 /$ rdlc. 916

Ce document a été généré automatiquement le 1 mai 2019.

\section{(c) (1) $\Theta \Theta$}

Recherches en didactique des langues et des cultures is licensed under a Creative Commons AttributionNonCommercial-NoDerivatives 4.0 International License 


\title{
La récapitulation en activité d'expression orale : entre complexité et autonomie
}

\author{
Farid Mazi
}

\section{Introduction}

1 Les travaux sur la réflexivité de la parole en contexte scolaire ont majoritairement ciblé le public enseignant afin de développer sa compétence professionnelle. L'ouvrage collectif intitulé Discours d'enseignants sur leur action en classe: Enjeux théoriques et enjeux de formation (Bigot et Cadet) fait foi d'une référence très représentative relative à l'actualité des recherches sur l'action didactique. Perrenoud aligne dans ce type de réflexivité ce que l'enseignant a réellement fait ou tenté de faire sans y parvenir: "la réflexion est à dominante rétrospective lorsqu'elle survient à l'issue d'une activité ou d'une interaction "; son rôle est de dresser un bilan et de préparer aux actions à venir(2001: 35). En revanche, nous envisageons dans ce texte de nous retourner plutôt vers l'apprenant afin de découvrir sa capacité à commenter son travail en classe avec des éléments linguistiques peu sophistiqués comparativement à ceux de son enseignant. Cicurel a précisé dans sa définition du répertoire didactique que ce dernier est le produit de la rencontre de plusieurs modèles didactiques parmi lesquels l'élève que l'on a été (2007 : 20). Il est de ce fait tentant de supposer que sa construction est ancienne et débute avec les premiers apprentissages. L'hypothèse qui en découle est qu'en classe, nous assistons au contact du répertoire de l'enseignant et celui de ses apprenants qui se partagent une jurisprudence didactique que justifient l'exercice et la manipulation en classe de la langue française. En étant habilités à faire des auto-commentaires sur leur faire et dire, nous supposons l'existence chez les apprenants d'un répertoire d'apprentissage qui leur permet de mettre en mots leur action passée. Pour illustrer notre propos, nous choisissons pour indicateur les séquences de récapitulation qui adviennent à la fin des activités orales. Elles servent en général à vérifier le résultat du travail réalisé durant toute la leçon. Ces activités 
résumantes sont motivées par l'idée de récapituler individuellement le contenu d'une interaction géré collectivement. Elles pourraient être assimilables à la phase de préclôture sans espérer forcément l'insertion d'actes précédant la fin des échanges. Étant la première partie de la clôture ratifiée, la pré-clôture offre selon Berthoud et Mondada, « la dernière possibilité d'insérer des mentionnables qui n'auraient pas eu l'occasion d'être développés dans le reste de la conversation "(1995: 281). Dans tous les cas de figure, demander à des apprenants de récapituler la leçon constitue une tâche exigeante au plan linguistique pour un bon nombre d'entre eux. Il s'agit pourtant d'une occasion d'apprentissage à saisir pour emprunter l'expression de Cicurel car les apprenants "savent s'emparer d'occasions pour prendre et reprendre la maîtrise de leur apprentissage » (2011:47).

\section{Approche actionnelle et répertoire (s) d'apprentissage}

L'approche de l'action concilie selon Cicurel et Rivière deux versants :

L'un plus interactionnel et qui s'attache aux pratiques effectives d'un sujet incarnant un rôle social, et l'autre plus enfoui, mais qui apparaît nettement lorsqu'on interroge l'acteur professeur et qu'il évoque les motifs sous-jacents au discours $(2008: 266)$.

3 Si cette approche est intrinsèquement orientée vers l'enseignant, elle peut fort bien s'adapter aux apprenants. Ces derniers, en tant que partenaires incontournables de l'agir en classe, interagissent au moment de la leçon mais ont quelque chose à dire après la clôture de l'interaction. Au même titre que leur enseignant, ils gardent des souvenirs de l'action passée et ils peuvent apporter des appréciations avantageuses sur le déroulement de la leçon. Leur répertoire d'apprentissage leur accorde justement ce droit légitime à la parole a posteriori que nourrissent leurs représentations et leurs acquis antérieurs. Ce répertoire n'est donc en rien une page vierge sur laquelle l'enseignant teste la didactisation de son savoir. C'est un palimpseste d'expériences qui déborde sur la frontière des connaissances académiques reçues dans l'espace de l'institution et qui s'attelle davantage à un savoir plus développé lié au contact avec l'entourage de la classe dont le principal catalyseur est l'interaction verbale. L'approche actionnelle ne devrait pas rester reléguée dans une réflexivité unilatérale qui ignore l'investissement discursif de l'apprenant. La situation de classe, pour autant qu'elle soit fondamentalement celle d'un enseignement/ apprentissage d'une langue étrangère, approuve aussi la rencontre d'un formateur avec des formés qui véhiculent des images et des stéréotypes centrés sur une action dont les interrelations entre savoir en interaction, un rôle enseignant et un rôle enseigné sont complexes. Morin indique dans sa réflexion sur l'action, que celle-ci « suppose la complexité, c'est-à-dire aléa, hasard, initiative, décision, conscience des dérives et des transformations » (2005: 107).Cette situation didactique fait émerger l'idée que dans la reformulation de notre approche de l'action, elle se situe à mi-chemin entre la parole de l'apprenant qui s'exprime avec ou sans l'étayage de l'enseignant et le potentiel linguistique mais surtout cognitif qui lui assurent l'interprétation de cette parole.

\section{Contexte de la recherche}

4 Dans le lycée, l'oral n'avait pas droit de cité avant 2005. Au primaire, son enseignement était confiné dans une progression linéaire dédiée à la phonétique articulatoire à raison de dix minutes la séance. Force est de reconnaître que les représentations des 
enseignants que confirment leurs pratiques de classe paraissent accorder une quasilégitimité à cet état des lieux. Elles désarment l'oral face à l'écrit en omettant de lui restituer sa place méritoire en qualité de vecteur vital de tout savoir et allié idéal qui garantit la réussite de l'action didactique, laquelle, selon les termes de Sensevy, renvoie à ce que " les individus font des lieux (des institutions) où l'on enseigne et où l'on apprend " (2007: 14). Sa réhabilitation dans les nouveaux programmes (2005) lui a permis de conquérir ses lettres de noblesse en faisant l'objet d'une formation fondée sur l'articulation, la compréhension, la production de messages oraux et l'interaction verbale. La valorisation de l'interaction se traduit surtout par le passage d'une relation frontale imposant une communication magistrale à une relation cohabitant avec des interactions de type enseignant-élève et élève-élève (Ferhani, 2006: 15). Comment ne pas admettre qu'il s'agisse d'un renouveau lorsque nous constatons de très près que les activités orales bénéficient au primaire et au collège d'un volume horaire concurrent à celui de l'écrit :

À l'école, les prescriptions du nouveau programme se traduisent par la quasiéquivalence en volume et en activités de l'oral et de l'écrit et permettent aujourd'hui d'exposer l'apprenant à des documents sonores authentiques (enregistrements audio d'émissions, chanson, discussion...) ouvrant à la découverte des variantes orales de la langue, à la meilleure compréhension des accents (id. : 16).

\section{Protocole d'expérimentation}

5 La leçon d'expression orale a eu lieu dans une classe de « FLE(Français Langue Étrangère) »de première année secondaire composée de 34 élèves dans un lycée situé à la périphérie de la ville d'Annaba en Algérie. Les interactions ont été filmées par nos soins en qualité d'observateur non participant. L'enseignante a présenté à cette occasion une activité sur le thème de la tricherie en situation d'examen dont le support figure sous la forme d'une bande dessinée. L'objet de cette expérimentation consiste d'abord à faire découvrir aux apprenants le thème global de la leçon et ses multiples étapes avant de leur demander de faire un récapitulatif et de déterminer leurs postures discursives et ressources d'apprentissage. Après cette phase de pré-clôture, l'observateur indiqué par l'abréviation $\mathrm{Ch}$, incarne le rôle d'interviewer et intervient auprès des apprenants ayant pris la parole afin de les interroger mais sans le recours à la méthode de l'auto-confrontation avec le film de leur prestation. Il leur demande s'ils ont résumé la leçon en s'appuyant sur les notes portées sur le tableau que voici :

- Le thème abordé c'est le copiage.

- Le copiage est une mauvaise action (fraude).

- Les raisons qui poussent l'élève à tricher :

- Le poids des leçons (l'élève est dépassé);

- L'élève a des difficultés d'apprentissage ;

- Un bon élève peut copier pour obtenir de bonnes notes (concurrence).

\section{Analyse des données}

6 Voici l'intervention principale de l'interviewer à l'issue de la leçon d'oral :

251.Ch : je vous remercie aussi mais je veux je voudrais comme même saisir l'occasion pour vous poser comme ça quelques questions par exemple le jeune-là qui est en face de moi ah enfin c'est des questions que je n'ai pas déjà préparées à l'avance c'est dans le tas donc je vous mets un peu en 
situation l'objectif en fait de cette expérimentation c'est de savoir si l'élève est capable de résumer une leçon d'oral qui est la leçon d'o $\boldsymbol{\ell}$ d'oral elle est un peu difficile parce que c'est pas comme à l'éc $\boldsymbol{\ell} l$ 'écrit d'accord euh encore est-ce que quand vous récapitulez ou quand vous résumez vous avez besoin par exemple de de de de certains

252. Pr: d'avoir des notes par exemple

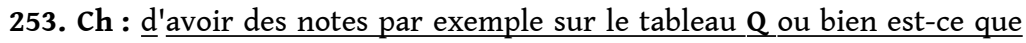
vous pouvez compter sur votre mém Cmémoire ou comment vous faites exactementQ

\section{Séquence récapitulative $n^{\circ} 1$}

\section{Une interaction entre ressources schématiques et schéma facilitateur}

216. Pr : est-ce que avez est-ce que vous avez saisi tout ce qu'on vient de faire Q- amh - allez-y - essayez de récapituler ce qu'on a di : $t$ tout à fait l'une des deux ce qu'on d'expliquer maintenant ce que je viens d'expliquer vous venez de trouver allez réfléchissez je vous donne un petit moment allez-y je vais vous aider bon la première partie on l'a récapitulée tout à l'heure alors ce que je viens de dire on ne doit pas tolérer ce genre de comportement au contraire on doit l'éradiquer d'accord le copiage est une tare c'est un défaut d'accord il diminue euh il diminue la la valeur la valeur de notre travail c'est une c'est c'est c'est une attitude immorale et tout cela euh a euh tout cela c'est un fléau plutôt qui favorise la médiocrité l'incompétence et tôt ou tard on va tomber dans la décadence et on va échouer dans notre vie que ce soit notre vie scolaire à commencer par le copiage donc on va échouer tôt ou tard allez-y oui

217. F (Chahira) : le thème abordé c'est le copiage c'est une mauvaise action euh qui a toujours existé non seulement dans le domaine scolaire ou universitaire ah universitaire la raison qui pousse l'élève àtricher c'est pour avoir des

218. Pr :de bonnes notes

219. F (Chahira) : des bonnes notes ou des élèves qui qui sont dépassés

220. Pr : oui

221. F (Chahira) : qui peuvent pas mémoriser ou par compétence avec deux bons deux bons é $\boldsymbol{\ell}$

222. $\operatorname{Pr}$ : concurrence

223. F (Chahira) : donc pour concurrence pour deux élèves voilà

224. Pr : c'est la concurrence qui le pousse à tricher pour avoir de bonnes

225. F (Chahira) :de bonnes notes

226. Pr : oui

227. F (Chahira) : et on pe $\boldsymbol{C}$ il faut pas amh

228. Pr : tolérer

229. F (Chahira) : tolérer ce cette action parce que c'est un défaut qui va nous ne nous $[\mathrm{m}$ i $\mathrm{n}$ e] tôt ou tard à la décadence

7 L'enseignante sollicite les apprenants afin de récapituler la leçon en leur proposant un schéma facilitateur : "comme dans toute interaction ritualisée, les participants suivent un scénario ou script, entendu comme une suite de comportements langagiers et corporels prévisibles » (Cicurel, 1994 :104). Néanmoins, pour réduire la difficulté de la tâche, Chahira priorise les ressources schématiques, c'est-à-dire des données prêtes à l'actualisation portées sur le tableau en commençant par évoquer dans l'ordre : le thème, le fait que la tricherie soit une mauvaise action et l'une des raisons qui poussent à accomplir cet acte, à savoir l'obtention de bonnes notes. Après avoir sécurisé son discours dès sa première prise de parole, elle s'ouvre sur le schéma de l'enseignante à partir du 
tour 227. Il semble que l'épuisement des données scripturales justifie ce recours à l'alternance du code écrit et oral chez l'apprenante afin de compléter son intervention: tolérer ce cette action parce que c'est un défaut qui va nous ne nous [ $\mathrm{m}$ i n e] tôt ou tard à la décadence. Nous constatons que ce segment est repris en grande partie du schéma d'interaction de l'enseignante dont voici les passages qui l'attestent :

- on ne doit pas tolérer ce genre de comportement;

- c'est un défaut ;

- tôt ou tard on va tomber dans la décadence.

\section{Commentaire de l'apprenante}

277. Ch : la fille qui est là Selam où est-ce qu'elle est Selma $Q$ où $\downarrow$

278. F (Selma) : je peux compter sur moi mais des fois je j'aime avoir des notes pour qui pour qu'elles pour qu'ils m'aident

279. Ch : voilà comment elle s'appelle $Q$

280. $\operatorname{Pr}$ : Chahira

281. Ch : Chahira voilà

282. F (Chahira) : des fois ça nous aide pour récapituler moi je ne compte pas sur ma mémoire

8 De par la réponse fournie par Chahira en 282, il ressort que le soutien principal qu'elle reçoit dans sa démarche de récapitulation relève prioritairement des ressources scripturales, voire la trace écrite sur le tableau et exclut l'atout que pourraient représenter les efforts cognitifs basés sur la mémoire. C'est ce qui explique par ailleurs la faible présence de données liées au cadrage de l'interaction par l'enseignante. Nous retenons à ce niveau un premier cas de posture discursive corrélée au répertoire d'apprentissage qui est d'ordre interactif-prescriptif.

\section{Séquence récapitulative $n^{\circ} 2$}

\section{Ressources schématiques}

230. Pr : très bien bien allez quelqu'un d'autre hein Houssem

231. G Houssem : le thème abordé c'est le copiage qui est considéré qui une mauvaise action considéré comme une fraude la la raison qui pousse ces élèves à tricher c'est pour avoir de bonnes notes pour passer à une autre classe ou par simple concurrence $\mathbf{X X}$ rivalité avec d'autres élèves du côté cette action est très mauvaise action qu'il faut supprimer de tous les établissements que ce soit scolaires ou universitaires ah

232. Pr : bien

233. G (Houssem) : et : c'est tout madame $\downarrow$

La seule intervention de l'apprenant, bien que longue, imite à quelques nuances près la structure des données schématiques et n'apporte pas d'éléments nouveaux qui ouvriraient le débat sur le thème du copiage. Houssem y met lui-même terme en 233 , ce qui montre le caractère limitatif de son intervention et la mise à l'écart du schéma fourni par l'enseignante. En termes plus clairs, il a fait une simple lecture de la trace écrite sur le tableau.

\section{Commentaire de l'apprenant}

269. $\mathrm{Ch}$ : on qui a aussi récapitulé $\mathbf{Q}$ comment il s'appelle $\mathbf{Q}$

270. $\mathrm{Pr}$ : Houssem

271. Ch : Houssem oui 
272. G (Houssem) : je peux compter sur moi mais je préférais avoir des notes sur le tableau

273. $\mathrm{Ch}$ : quelques notes des repères sur le tableau oui Autrement énoncé, bien que sa capacité à récapituler la leçon soit évidente pour lui, il a avantagé les notes du tableau. De ce fait, il fait montre d'une posture strictement prescriptive vis-à-vis de la tâche qui lui a été confiée, ce qui n'enrichit pas son répertoire d'apprentissage.

\section{Séquence récapitulative $n^{\circ} 3$}

\section{Ressources schématiques}

234. Pr : bien quelqu'un d'autre allez-y allez Mohamed amh

235. G (Amir) : le thème abordé dans ce texte c'est le copiage c'est qui est mauvaise action que qu'il faut supprimer dans le domaine universitaire et les raison qui poussent l'élève à tricher c'est c'est c'est la concurrence par rapport à aux élèves aux bons élèves et et et les élèves trichent pour avoir de bonnes notes et il y a des élèves qui avaient des difficultés à à apprendre

236. $\operatorname{Pr}$ : chut chut qui ont des diffi $\boldsymbol{\ell}$ difficultés

237. G (Amir) : qui ont des difficultés à apprendre euh c'est une action immorale

11 À la lueur de l'intervention de Amir en 235, il ressort qu'il a respecté à la lettre la structure des données schématiques. Plus clairement, il est question d'une quasi-lecture des notes portées sur le tableau et l'apprenant ne semble pas faire grand cas du schéma circonstancié qui a été proposé par l'enseignante. Aucun effort d'innovation n'a été consenti pour introduire de nouveaux éléments à même d'étoffer son récapitulatif. À l'instar de ses deux camarades, nous retrouvons les mêmes préliminaires d'ouverture comme celle du thème abordé.

\section{Commentaire de l'apprenant}

261. $\mathrm{Ch}$ : très bien ah ah comment il s'appelle $\mathbf{Q}$

262. Pr : Amir

263. Ch : Amir Amir oui est-ce que tu peux tu peux répondre à ma question $\mathbf{Q}$ est-ce que tu as besoin des notes qui sont portées sur le tableau pour récapituler $\mathbf{Q}$

264. G (Amir) : oui ah on on

265. $\mathrm{Ch}$ : est-ce que tu en as besoin est-ce que tu éprouves le besoin d'avoir des notes sur le tableau pour récapituler ce qu'on a expliqué oui ou non $\mathbf{Q}$

266. G(Amir) : oui j'ai vraiment besoin de ces notes

La brièveté de la réponse de l'apprenant en 266 est totalement compatible avec la façon dont il a récapitulé la leçon. C'est une réponse réactive qui dénote tout simplement du besoin insistant des notes portées sur le tableau d'où leur lecture sans possibilité de reformulation ou d'ajout. L'apprenant adopte de ce fait une posture strictement prescriptive qui ne laisse aucune chance à l'innovation ou au perfectionnement de son répertoire d'apprentissage. Il est tout aussi probable d'affirmer que les lacunes en langue et le fait d'interroger pour la première fois le répertoire des trois élèves interviewés n'ont pas facilité l'établissement d'une autoscopie pointue de leur réflexivité. En conséquence, cette analyse recommanderait la mise sur pied de nouvelles expérimentations afin de sonder la zone des non-dits présents dans leurs discours rétrospectifs. 


\section{Séquence récapitulative $n^{\circ} 4$}

\section{Ressources expansives}

238. Pr : amh hein bien quelqu'un d'autre vas-y

239. G (Mohamed) : le copiage la fraude est une action immorale qui mène à la décadence dans la société et de la personne elle-même cet acte cet acte ah pousse

240. Pr : chut

241. G (Mohamed): ah eh eh pousse beaucoup d'él $\boldsymbol{C}$ pousse à l'échec scolaire ou même dans la vie active ah beaucoup d'él $\boldsymbol{e b e a u c o u p ~ d ' e ́ l e ̀ v e s ~ e t ~}$ en majorité peut font des pratiques de la triche pendant les examens ou les tests car le poids des leçons est beaucoup trop lourd ou ils ont des difficultés ah d'apprentissage et il y a aussi de bons élèves qui qui sont poussés par la rivalité et la concurrence vis-à-vis aux à leurs pairs de faire de par $\varnothing$ pratiquer le copiage ou la triche pour avoir de meilleures notes

242. Pr : amh

243. G (Mohamed) : ce copiage

244. Pr : ce comportement oui

245. G (Mohamed) : ce copiage donnera des éléments faibles ou même médiocres dans la société future ce qui mènera à l'échec de toute une nation 246. Pr : bien : $\mathrm{n}$ très bien

247. G (Mohamed) : cet acte doit être réprimandé et supprimé par toutes autorités par des sanctions plus au moins gra $\ell$ plus ou moins sévères comme dans les examens [ $\mathrm{f} i] \rightarrow$ dans comme dans les les examens comment dire officiels dans l'exemple du baccalauréat donc on prend quelqu'un en train de tricher il est sanctionné par cinq ans sans passe le l'examen

248. Pr : oui

249. G (Mohamed) : ce genre d'acte doit être plus présenté dans les éta $\boldsymbol{C}$ les établissements scolaires par exemple même dans un examen non officiel de trimestre doit être sanctionné par un renvoi nous donnerons beaucoup d'idées dans la prochaine séance

250. Pr : très bien Mohamed il a trouvé des solutions au problème ou à ce fléau très bien très bien

13 La longueur des interventions de Mohamed prouvent la maîtrise de son discours et sa capacité à dépasser les données schématiques ainsi que le schéma proposé par l'enseignante. Les énoncés qu'il a formulés sont de loin mieux expressifs que ceux de ses pairs et se distinguent favorablement par l'apport de solutions prometteuses au phénomène du copiage. Son discours ne reproduit pas la structure des notes portées sur le tableau et ne démarre pas son propos en réitérant comme dans les trois cas précédents la formule leitmotiv le thème abordé c'est, etc. Le caractère innovant de son discours se remarque particulièrement dans le détournement même de l'étayage de l'enseignante au niveau du tour 244 alors qu'il n'était pas en panne d'inspiration : ce comportement oui. L'apprenant reprend le terme qu'il a utilisé dans le tour 243 et le réinvestit une deuxième fois dans la suite de son intervention. Autre spécificité de ce récapitulatif, c'est qu'il n'a été émaillé que par deux régulateurs d'écoute et aucun signe de soutien professoral n'a été enregistré. Il constitue en l'occurrence un cas typique d'un résumé réussi comme le reconnaît l'enseignante à la fin :très bien Mohamed il a trouvé des solutions au problème ou à ce fléau très bien très bien.

Commentaire de l'apprenant 
254. G (Mohamed) : personnellement je n'utilise pas les notes du tableau

255. Ch : comment comment il s'appelle $\mathbf{Q}$

256. G (Mohamed) : Mohamed Anis

257. Ch : oui

258. G (Mohamed) : personnellement je n'utilise pas les notes dans tableau j'essaie de mémoriser dans ma tête et puis

259. $\mathbf{C h}$ : bien très bien ah ou tu veux continuer $\mathbf{Q}$

260. G (Mohamed) : les idées se mêlent entre-elles puis j'ai des idées c'est simple

14 Nous remarquons une nette compatibilité entre la démarche suivie par l'apprenant Mohamed et son auto-commentaire. Il se réapproprie son action en usant à deux reprises $\mathrm{du}$ modalisateur personnellement et insiste sur le fait qu'il écarte toute exploitation des notes du tableau. Le volume de son récapitulatif et les éléments qu'il a injectés dans son intervention semblent être une prouesse de sa mémorisation. Il admet qu'il table plus sur la structuration cognitive des idées qu'il finit par verbaliser en adoptant une posture magistrale qui rend compte d'un répertoire d'apprentissage dont les ressources sont de nature expansive.

\section{Bilan de l'analyse des séquences}

De par l'analyse réalisée des séquences récapitulatives et des auto-commentaires respectifs, notamment l'aisance avec laquelle s'est exprimé le dernier apprenant, nous avons dégagé deux conditions sine qua non, voire deux aspects. Ils assurent l'accomplissement d'une tâche aussi contraignante que le résumé d'une leçon d'expression orale par des apprenants disposant d'un répertoire d'apprentissage qui exige le passage d'une série d'expériences afin de définir ses besoins et ses limites tout en apportant des réflexions sur leur action passée. Dans le même sillage, il est pertinent de considérer la récapitulation effectuée par l'apprenant de cruciale pour la capitalisation et la mise à l'épreuve des connaissances inhérentes au processus de son apprentissage. Il peut, selon ses moyens linguistiques, opérer en autonomie totale, en semi-autonomie ou sous l'égide du professeur qui le guide dans sa production verbale.

\section{a/ D'abord la complexité}

Selon les termes de Morin, la complexité est entendue comme "un phénomène quantitatif, l'extrême quantité d'interactions et d'interférences entre un très grand nombre d'unités " (2005: 48). C'est pour cela que nous considérons les séquences récapitulatives comme une opération complexe car elle requiert l'investissement de l'ensemble des compétences dont le sujet parlant dispose dans la langue étrangère et surtout la compétence à communiquer oralement. L'apprenant-interactant s'inscrit dans un cadre participatif qui le transforme en acteur qui s'apprête à accomplir cette tâche en étant conscient de toute sa complexité. De surcroît, il devient l'auteur incontestable des ses dires dans le projet de récapitulation.

\section{b/ Ensuite l'autonomie}

Pouvoir récapituler c'est aussi se donner les moyens idoines pour s'autonomiser vis-à-vis du dispositif de soutien installé en classe et ce pour éviter de restituer à la lettre un contenu déjà existant. Il devrait faire office d'un canevas pour reconfigurer puis innover en adoptant une posture discursive magistrale. Mais cette autonomie, pour autant qu'elle soit l'œuvre du recul observé à l'égard de ces moyens, est tout autant sous réserve. Elle 
demeure tributaire des ressources inavouées au moment de la prestation. En tant que notion, cette autonomie rappelle Morin «se nourrit de dépendance; nous dépendons d'une éducation, d'un langage, d'une culture, d'une société » (id. : 89). Ce sont ces ressources qui font de l'apprenant le maître du jeu discursif qui rend ses dires cohérents quel que soit le projet didactique à mettre en scène.

\section{Conclusion}

Ce texte représente une ébauche d'un projet qui a besoin d'être réexpérimenté avec d'autres classes et d'autres niveaux pour élargir la recherche sur les postures discursives. Il inaugure une nouvelle génération de travaux en FLE qui aborde la problématique de la récapitulation en activité d'expression orale mais en particulier celle de la confrontation des apprenants avec leur action dans le but de la commenter et prendre plus conscience de leur répertoire d'apprentissage. Les différentes postures que nous avons délimitées mettent en relief les écarts flagrants dans la qualité et la quantité des informations d'une part, et aussi les ressources qui les inspirent pour mener à bien cette tâche. Nous avons constaté à quel point la concision des commentaires même celle en adéquation avec la posture magistrale est insuffisante pour dresser des bilans probants qui font dégager et décrire les ressources principales ayant conduit les apprenants à afficher un tel comportement langagier. À l'évidence, il paraît plus concordant d'établir à l'avenir une auto-confrontation simple ou croisée des apprenants avec le film de leur prestation. Visiblement, cette technique d'investigation est capable de tirer le maximum de renseignements sur les motifs qui les engagent à assumer une posture discursive en conformité avec leur savoir-faire. Nous pouvons découvrir dans quelle mesure il est impossible pour certains d'entre eux d'empiéter sur les données schématiques ou l'étayage interactionnel du professeur tandis que d'autres font preuve d'une flexibilité verbale qui dépasse le contenu pré-construit nécessitant tout au plus un effort de reformulation ou de lecture littérale. La question posée dans cette recherche concerne en premier chef les éléments à développer par des apprenants qui ne sont ni sollicités ni ayant pris l'initiative de participer pendant le cours. Il sied d'entreprendre une sorte de compétition au sein du groupe-classe qui permettrait d'identifier des postures inaccessibles quand une bonne partie du public préfère rester en expectative. Autant avouer en toile de fond qu'une action didactique intelligente devrait procurer une base de données plus intéressante au chercheur dès lors où elle commence par cibler en priorité les apprenants qui n'agissent pas spontanément faute d'une compétence communicative faisant écho aux exigences didactiques de la récapitulation d'une leçon d'oral.

\section{BIBLIOGRAPHIE}

Berthoud, A.-C. \& Mondada, L. (1995). « Mode d'introduction du topic dans l'interaction verbale ». In Véronique, D.\& Vion, R. (coord.).Modèles de l'interaction verbale. Université de Provence : PUP. 
Bigot, V.\& Cadet, L. (2011). Discours d'enseignants sur leur action en classe :Enjeux théoriques et enjeux de formation. Paris : Riveneuve Édition.

Cicurel, F.(1994). « Schéma facilitateur et métalangage dans l'apprentissage d'une langue étrangère ».In Lapeyre, F.(coord.).Cahier du français contemporain 1. Simple-Simplification. Paris : Didier Érudition.

Cicurel, F.(2007). « L'agir professoral, une routine ou une action à haut risque ». In Plazaola Giger, I.\& Stroumaza, K. (éds.). Parole de praticiens et description de l'activité. Problématisation méthodologique pour la formation et la recherche. Bruxelles : De Boeck Université.

Cicurel, F.\& Rivière, V. (2008). « De l'interaction en classe à l'action revécue : le clair-obscur de l'action enseignante ». In Filliettaz, L.\& Schubauer Leoni, M.-L. (éds.). Processus interactionnels et situations éducatives. Bruxelles : Édition De Boeck Université.

Djilali, K. (2005). Direction de l'enseignement secondaire, commission nationale des programmes. Alger : ONPS.

Ferhani, F.-F. (2006). « Algérie, l'Enseignement du Français à la Lumière de la Réforme ». Le Français aujourd'hui, ${ }^{\circ} 154$, pp. 11-18.

Morin, E. (2005). Introduction à la pensée complexe. Paris :Édition du Seuil.

Perrenoud, P. (2001). Développer la pratique réflexive dans le métier enseignant. Paris : ESF Éditeur.

Sensevy, G. (2007). « Des catégories pour décrire et comprendre l'action didactique ». In Sensevy, G.\& Mercier, A. (dir.).Agir ensemble : l'action didactique conjointe du professeur et des élèves. Rennes : PUR.

\section{ANNEXES}

\section{Notes}

Convention de transcription de l'équipe de GARS (Groupe Aixois de Recherche en Syntaxe et Analyse Discursive) : Majuscule pour les noms propres - Interruption ou coupure de la bande ///- séquence incompréhensible : XXX - Chevauchement Multitranscription /........./ - Commentaire de l'observateur : (......) - Amorce d'un mot + Discours rapporté » « - Syllabation : mer...cre.....di. (Blanche-Benveniste, 1997 : 34).

Signes ajoutés : $\varnothing$ (inachèvement d'un mot)- Q (question) -Pr : Professeur - G : Garçon - F : Fille - F/G ( ? ) : élève non identifié- Aps : Plusieurs élèves - $\mathrm{Ch}$ : interviewer.

L'enseignante qui a présenté la leçon tenait à ce que son nom figure dans l'article. Elle s'appelle Amel Ait Kaki et possède à son actif plus de 16 ans d'expériences dans l'enseignement de la langue française (primaire et secondaire). Elle a obtenu une licence de 4 ans d'étude en langue et lettres françaises de l'université Sidi Amar à Annaba en 1999.

\section{RÉSUMÉS}

Notre contribution se veut une esquisse d'un travail qui permet de bien comprendre le déroulement de l'activité de récapitulation et son rapport avec la prestation des apprenants. Dans notre texte, nous avons essayé de jeter les amorces d'une recherche qui vise à connaître les 
postures discursives adoptées par des lycéens algériens lors des séquences récapitulatives. Nous avons mis en adéquation leur capacité à récapituler une leçon d'oral avec la verbalisation des ressources sur lesquelles ils s'appuient pour venir à bout de cette tâche. L'analyse qui a été réalisée a permis de retenir deux conditions associées à cette tâche : la complexité de l'opération et l'autonomie dans la gestion des ressources du répertoire d'apprentissage.

Our contribution requires a sketch of a work which permits a good understanding of the summarizing activity in progress and its relation to learners with their performance. In our text, we have tried to lay the primers unprecedented research to know the postures adopted by the Algerian high school students during the summary sequences to align their ability to summarize an oral expression lesson with the word development resources on which they rely to overcome the task. The analysis which was carried out allowed us to retain two conditions associated to this task: the complexity of the operation the autonomy in the management of learning resources repertory.

\section{INDEX}

Mots-clés : séquences récapitulatives, approche actionnelle, répertoire d'apprentissage, complexité, autonomie

Keywords : summary sequences, action-oriented approach, learning directory, complexity, autonomy

\section{AUTEUR}

\section{FARID MAZI}

Université Badji-Mokhtar-Annaba. Algérie

Adresse : Rue Tahar Saad Saoud. Villa 127 F. Les Caroubiers. Annaba 23000. Algérie

Farid Mazi est maître de conférences en didactique du FLE au département de français de la Faculté des Lettres, Sciences Humaines et Sociales, El-Bouni/Annaba. Ses recherches intéressent essentiellement la formation du corps professoral, l'action de l'enseignant et les interactions verbales en classe de français langue étrangère.

Courriel :farid.mazi[at]yahoo.fr 